\title{
Synthesis and antibacterial activity of a series novel 5,7-diisoprenyloxyflavone derivatives
}

\author{
Qing-Hao Jin ${ }^{\circledR *}$, Zhi-Yang Fu², Ya-Nan Xia², Bing-Yu Liu², Hai-Ying Jiang ${ }^{3 *}$ \\ ${ }^{1}$ Donghai Science and Technology College, Zhejiang Ocean University, Zhejiang, Zhoushan, P. R. China, ${ }^{2}$ Food and Pharmacy \\ College, Zhejiang Ocean University, Zhejiang, Zhoushan, P. R. China, ${ }^{3}$ College of medicine, Jiaxing University, Zhejiang, \\ Jiaxing, P. R. China
}

\begin{abstract}
In the present study, a series of novel 5,7-diisoprenyloxyflavone derivatives were designed, synthesized, and evaluated for their antibacterial activity. Most of these compounds displayed significant antibacterial effects against Gram-positive bacteria, especially against strains of multidrug-resistant clinical isolates. Compounds $\mathbf{4 c}, \mathbf{4 g}, \mathbf{4 i}, \mathbf{4 j}, \mathbf{4 k}, \mathbf{4}, \mathbf{4 n}, \mathbf{4 q}$ and $\mathbf{4 t}$ showed high levels of antimicrobial activity against Staphylococcus aureus RN4220 with minimum inhibitory concentrations of 4.0-20 $\mu \mathrm{M}$. Compound $4 \mathbf{k}$ showed the most potent activity among these compounds against all multidrug-resistant clinical isolates tested. Unfortunately, none of the compounds were active against Gram-negative bacteria at the doses of $24-164 \mu \mathrm{M}$.
\end{abstract}

Keywords: Flavonoids. Synthesis. Isoprenyloxyflavones. Antibacterial activity.

\section{INTRODUCTION}

According to the World Health Organization (WHO), infectious diseases are responsible for a significant proportion of deaths worldwide, with antimicrobial agents considered "miracle drugs" that are the leading weapons in the treatment of infectious diseases. However, owing to the development of antimicrobial resistance and the appearance of drug-resistant strains among communityacquired infections, there is evidence of the rapid global spread of resistant clinical isolates, with many current clinically efficacious antimicrobial agents becoming less effective. Therefore, the treatment of bacterial infections remains an important and challenging therapeutic problem (Chen et al., 2010). Ongoing drug discovery is necessary for identifying effective, safe, and affordable cures for an expanding spectrum of human ailments. As the treatment of these diseases has serious safety and efficacy issues, the exploration of new antibacterial agents is highly desirable.

Malaria caused by protozoan parasites of the genus plasmodium is a major cause of mortality and

\footnotetext{
*Correspondence: Q.H. Jin. Donghai Science and Technology College, Zhejiang Ocean University, 316000-Zhejiang, Zhoushan, P. R. China. E-mail: qhjin2012@163.com

H.Y. Jiang. College of Medicine, Jiaxing University, 314001-Zhejiang, Jiaxing, P. R.China. Tel: +86-580-13957204963. E-mail: jiangyang7689@aliyun.com
}

morbidity, especially in tropical countries. More than three billion people worldwide have been affected by this deadly disease (Verlinden, Louw, Birkholtz, 2016). The increasing resistance of malarial parasites to available drugs is a major reason for these large statistics, despite many reports on the antimalarial efficacy of flavonoids (Friedman, 2007; Kozyra et al., 2015). The development of novel antimicrobial drugs is still in demand owing to increasing incidence of infection caused by the rapid development of microbial resistance to most known antibiotics.

Flavonoids are natural products found throughout nature that have broad physiological activities, low toxicity, and few side effects (Harborne, Williams, 2000). Flavonoids are a diverse class of polyphenolic phytochemicals found in various vegetables and fruits, and provide color, aroma, flavor, and nutritional and health benefits (Karakaya, Sedef, 1999). Many flavonoids extracted from plants have been reported to show various biological effects, including anticancer, antioxidant, antibacterial, anti-inflammatory, and antidepressant effects (Ahmed, Khan, Saeed, 2015; Xie et al., 2014; Kulbacka et al., 2016; Yao et al., 2014; Keshari et al., 2016). Apigenin (5,7,4'-trihydroxyflavonoid; Figure 1), a bioflavonoid widely found in citrus fruits, has been found to exert a variety of pharmacological effects, 
including antibacterial, anticancer, and antiproliferative effects (Banerjee et al., 2015; Liu et al., 2013; Eumkeb, Chukrathok, 2013; Choi et al., 2010; Turktekin et al., 2011; Ruivo et al., 2015). The prenyl moiety is widely found in many drugs and natural products (Zhao et al., 2011; Winans et al., 1999). Prenylation has been reported to produce flavonoids with enhanced antibacterial, antioxidant, anti-inflammatory, larvicidal, cytotoxicity, and estrogenic activities (Rao et al., 2009; Vogel et al., 2008; Vogel et al., 2010). It has been proposed that prenyl moieties can make a compound more lipophilic, leading to a high affinity with cell membranes (Chen et al., 2014; Marín, Máñez, 2013; Coelho et al., 1992).

To obtain new compounds with better antibacterial effects, and as part of our ongoing research on structurebased design using apigenin as the lead compound, the introduction of a prenyloxy group on the A-ring of apigenin was used to prepare a series of 20 novel 5,7-diisoprenyloxyflavone derivatives (4a-4t; Scheme-1). These compounds were synthesized, characterized, and screened for their antibacterial activities. The synthesized of 5,7-diisoprenyloxyflavone derivatives $(\mathbf{4 a - 4 t})$ and antibacterial effects are not reported and is the originality compounds.

\section{MATERIAL AND METHODS}

\section{Chemistry}

Melting points were determined in open capillary tubes and were uncorrected. IR spectra were recorded on a FT-IR1730 (Bruker, Switzerland) using KBr pellets. ${ }^{1} \mathrm{H}-\mathrm{NMR}$ and ${ }^{13} \mathrm{C}$-NMR spectra were measured on an AV-300 spectrometer (Bruker, Switzerland), with all chemical shifts given in ppm relative to tetramethylsilane standard. High-resolution mass spectrometry (HRMS) was performed on an MALDI-TOF/TOF mass spectrometer (Bruker Daltonik, Bremen, Germany). Major chemicals were purchased from Aldrich Chemical Corporation (Shanghai, China), and all chemicals were of analytical grade. Compounds 1a-1t, 2a-2t and 3a-3t were synthesized to refer to previously published literature (Guan et al., 2013; Xie et al., 2014.).

\section{General procedure for the preparation of compounds (4a-4t)}

To a stirred solution of compounds $\mathbf{3 a}-\mathbf{3 t}(0.4 \mathrm{mmol})$ in DMSO $(30 \mathrm{~mL})$ in a $100-\mathrm{mL}$ round-bottomed flask was added $\mathrm{I}_{2}(0.4 \mathrm{mmol})$. The mixture was then stirred at $100-130{ }^{\circ} \mathrm{C}$ for $3-5 \mathrm{~h}$ to achieve reaction completion (as monitored by TLC) (Kim et al., 2007). The reaction mixture was then poured into ice-water to produce a yellow precipitate, which was collected and recrystallized from ethanol to afford corresponding products $4 \mathbf{a}-\mathbf{4 t}$. The yields and IR, ${ }^{1} \mathrm{H}-\mathrm{NMR},{ }^{13} \mathrm{C}-\mathrm{NMR}$, and mass spectral data for each compound are provided below.

\section{5,7-Diisoprenyloxyflavone (4a)}

Yield: 79.3\%; mp 74-76 ${ }^{\circ} \mathrm{C}$; ${ }^{1} \mathrm{H}-\mathrm{NMR}$ (DMSO- $d_{6}$, $300 \mathrm{MHz}): \delta 1.63\left(\mathrm{~s}, 3 \mathrm{H},-\mathrm{CH}_{3}\right), 1.66\left(\mathrm{~s}, 3 \mathrm{H},-\mathrm{CH}_{3}\right), 1.72$ $\left(\mathrm{s}, 3 \mathrm{H},-\mathrm{CH}_{3}\right), 1.76\left(\mathrm{~s}, 3 \mathrm{H},-\mathrm{CH}_{3}\right), 4.42\left(\mathrm{~d}, 2 \mathrm{H},-\mathrm{CH}_{2}\right)$, $4.57\left(\mathrm{~d}, 2 \mathrm{H},-\mathrm{CH}_{2}\right), 5.38(\mathrm{t}, 1 \mathrm{H},=\mathrm{CH}), 5.49(\mathrm{t}, 1 \mathrm{H},=\mathrm{CH})$, 6.09-6.12 (m, 2H, $\left.-\mathrm{C}_{6} \mathrm{H}_{2}\right), 6.81(\mathrm{~s}, 1 \mathrm{H},=\mathrm{CH}), 7.16-7.38$ $\left(\mathrm{m}, 5 \mathrm{H},-\mathrm{C}_{6} \mathrm{H}_{5}\right) ;{ }^{13} \mathrm{C}-\mathrm{NMR}$ (DMSO- $\left.d_{6}, 75 \mathrm{MHz}\right): \delta 19.7$, 20.1, 25.7, 26.0, 45.7, 45.9, 98.1, 99.7, 104.0, 105.3, 123.7, $124.0,126.7,127.0,128.3,128.9,129.3,130.7,132.5$, 132.8, 160.4, 163.3, 164.2, 168.7, 182.8; IR (KBr) cm $\mathrm{cm}^{-1}$ :

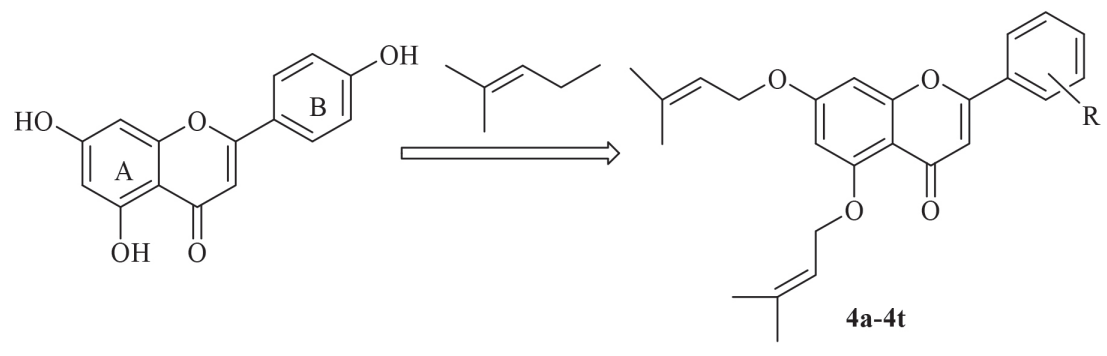

$$
\begin{array}{lllll}
\mathrm{R}: & & & \\
\mathbf{4} \mathbf{a}=\mathrm{H} & \mathbf{4} \mathbf{b}=2-\mathrm{F} & \mathbf{4} \mathbf{c}=3-\mathrm{F} & \mathbf{4 d}=4-\mathrm{F} & \mathbf{4 e}=2-\mathrm{Cl} \\
\mathbf{4 f}=3-\mathrm{Cl} & \mathbf{4} \mathbf{g}=4-\mathrm{Cl} & \mathbf{4 h}=2-\mathrm{Br} & \mathbf{4 i}=3-\mathrm{Br} & \mathbf{4 j}=4-\mathrm{Br} \\
\mathbf{4} \mathbf{k}=2-\mathrm{Cl}-4-\mathrm{Cl} & \mathbf{4}=4-\mathrm{NO}_{2} & \mathbf{4} \mathbf{m}=2-\mathrm{CH}_{3} & \mathbf{4 n}=3-\mathrm{CH}_{3} & \mathbf{4 0}=4-\mathrm{CH}_{3} \\
\mathbf{4} \mathbf{p}=2-\mathrm{OCH}_{3} & \mathbf{4 q}=3-\mathrm{OCH}_{3} & \mathbf{4} \mathbf{r}=4-\mathrm{OCH}_{3} & \mathbf{4} \mathbf{s}=4-\mathrm{N}\left(\mathrm{CH}_{3}\right)_{2} & \mathbf{4 t}=3-\mathrm{OCH}_{3}-4-\mathrm{OH}
\end{array}
$$

FIGURE 1 - The structure of apigenin and general structure of compounds $\mathbf{4 a - 4 t}$. 


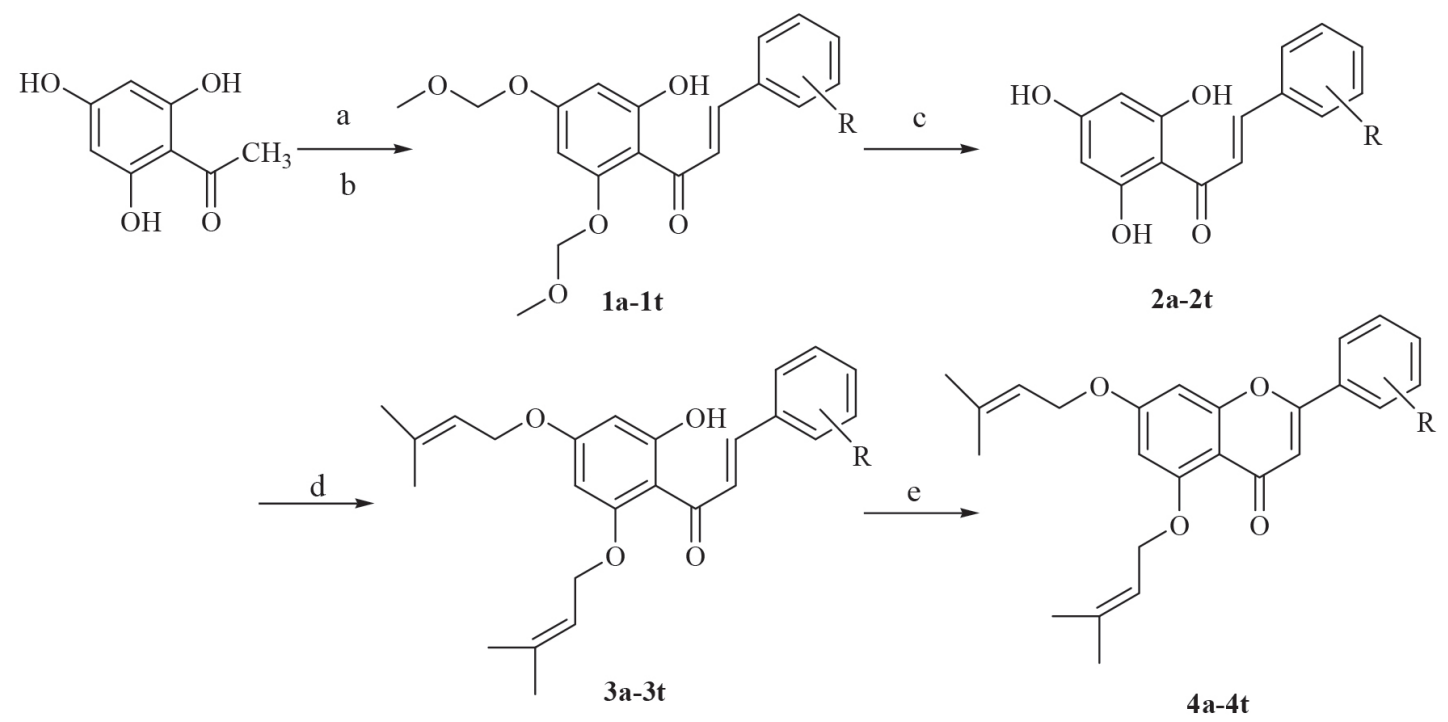

$\mathrm{R}$ :

$$
\begin{array}{lllll}
\mathbf{4 a}=\mathrm{H} & \mathbf{4 b}=2-\mathrm{F} & \mathbf{4 c}=3-\mathrm{F} & \mathbf{4 d}=4-\mathrm{F} & \mathbf{4 e}=2-\mathrm{Cl} \\
\mathbf{4 f}=3-\mathrm{Cl} & \mathbf{4 g}=4-\mathrm{Cl} & \mathbf{4 h}=2-\mathrm{Br} & \mathbf{4 i}=3-\mathrm{Br} & \mathbf{4 j}=4-\mathrm{Br} \\
\mathbf{4 k}=2-\mathrm{Cl}-4-\mathrm{Cl} & \mathbf{4 l}=4-\mathrm{NO}_{2} & \mathbf{4} \mathbf{m}=2-\mathrm{CH}_{3} & \mathbf{4 n}=3-\mathrm{CH}_{3} & \mathbf{4 o}=4-\mathrm{CH}_{3} \\
\mathbf{4} \mathbf{p}=2-\mathrm{OCH}_{3} & \mathbf{4 q}=3-\mathrm{OCH}_{3} & \mathbf{4 r}=4-\mathrm{OCH}_{3} & \mathbf{4 s}=4-\mathrm{N}\left(\mathrm{CH}_{3}\right)_{2} & \mathbf{4 t}=3-\mathrm{OCH}_{3}-4-\mathrm{OH}
\end{array}
$$

Reagents and conditions:

(a) $\mathrm{ClCH}_{2} \mathrm{OCH}_{3}, \mathrm{~K}_{2} \mathrm{CO}_{3}$; (b) aromatic aldehyde, $\mathrm{KOH}$; (c) $3 \mathrm{M} \mathrm{HCl}$; (d) prenyl bromide, anhydrous $\mathrm{K}_{2} \mathrm{CO}_{3}$; (e) $\mathrm{I}_{2}$, DMSO.

SCHEME1 - Synthesis routes of target compounds 4a-4t.

2931, 1678, 1221, 970; ESI-HRMS calcd. for $\mathrm{C}_{25} \mathrm{H}_{26} \mathrm{O}_{4}^{+}$ $\left([\mathrm{M}+\mathrm{H}]^{+}\right): 391.183$, found: 391.1820 .

\section{2'-Fluoro-5,7-diisoprenyloxyflavone (4b)}

Yield: $61.2 \%$; mp 92-94 ${ }^{\circ} \mathrm{C}$; ${ }^{1} \mathrm{H}-\mathrm{NMR}$ (DMSO- $d_{6}$, $300 \mathrm{MHz}): \delta 1.62\left(\mathrm{~s}, 3 \mathrm{H},-\mathrm{CH}_{3}\right), 1.65\left(\mathrm{~s}, 3 \mathrm{H},-\mathrm{CH}_{3}\right), 1.73$ $\left(\mathrm{s}, 3 \mathrm{H},-\mathrm{CH}_{3}\right), 1.74\left(\mathrm{~s}, 3 \mathrm{H},-\mathrm{CH}_{3}\right), 4.47\left(\mathrm{~d}, 2 \mathrm{H},-\mathrm{CH}_{2}\right)$, $4.61\left(\mathrm{~d}, 2 \mathrm{H},-\mathrm{CH}_{2}\right), 5.40(\mathrm{t}, 1 \mathrm{H},=\mathrm{CH}), 5.47(\mathrm{t}, 1 \mathrm{H},=\mathrm{CH})$, 6.05-6.11 (m, 2H, $\left.-\mathrm{C}_{6} \mathrm{H}_{2}\right), 6.67(\mathrm{~s}, 1 \mathrm{H},=\mathrm{CH}), 6.98-7.32$ $\left(\mathrm{m}, 4 \mathrm{H},-\mathrm{C}_{6} \mathrm{H}_{4}\right) ;{ }^{13} \mathrm{C}-\mathrm{NMR}$ (DMSO- $\left.d_{6}, 75 \mathrm{MHz}\right): \delta 19.9$, 20.3, 25.5, 25.9, 45.5, 45.8, 97.8, 98.9, 103.7, 104.8, $116.4,121.8,123.6,123.8,124.7,128.7,129.6,132.6$, $132.7,158.9,162.7,163.8,168.5,182.6$; IR $(\mathrm{KBr}) \mathrm{cm}^{-1}$ : 2934, 1676, 1220, 972; ESI-HRMS calcd. for $\mathrm{C}_{25} \mathrm{H}_{25} \mathrm{FO}_{4}^{+}$ $\left([\mathrm{M}+\mathrm{H}]^{+}\right): 408.1737$, found: 408.1742 .

\section{3'-Fluoro-5,7-diisoprenyloxyflavone (4c)}

Yield: $59 \%$; mp $98-100{ }^{\circ} \mathrm{C}$; ${ }^{1} \mathrm{H}-\mathrm{NMR}$ (DMSO- $d_{6}$, $300 \mathrm{MHz}): \delta 1.66\left(\mathrm{~s}, 3 \mathrm{H},-\mathrm{CH}_{3}\right), 1.69\left(\mathrm{~s}, 3 \mathrm{H},-\mathrm{CH}_{3}\right), 1.71(\mathrm{~s}$, $\left.3 \mathrm{H},-\mathrm{CH}_{3}\right), 1.73\left(\mathrm{~s}, 3 \mathrm{H},-\mathrm{CH}_{3}\right), 4.44\left(\mathrm{~d}, 2 \mathrm{H},-\mathrm{CH}_{2}\right), 4.69(\mathrm{~d}$, $\left.2 \mathrm{H},-\mathrm{CH}_{2}\right), 5.47(\mathrm{t}, 1 \mathrm{H},=\mathrm{CH}), 5.65(\mathrm{t}, 1 \mathrm{H},=\mathrm{CH}), 6.06-6.14$ $\left(\mathrm{m}, 2 \mathrm{H},-\mathrm{C}_{6} \mathrm{H}_{2}\right), 6.67(\mathrm{~s}, 1 \mathrm{H},=\mathrm{CH}), 6.82-7.28(\mathrm{~m}, 4 \mathrm{H}$, $-\mathrm{C}_{6} \mathrm{H}_{4}$ ); ${ }^{13} \mathrm{C}-\mathrm{NMR}$ (DMSO-d $6,75 \mathrm{MHz}$ ): $\delta 19.2,19.7$, 25.1, 25.5, 65.8, 66.0, 99.1, 100.1, 103.9, 105.3, 111.8, $115.1,122.8,123.4,123.7,130.4,132.4,132.8,133.0$, 159.6, 162.3, 162.7, 163.6, 168.4, 183.1; IR (KBr) cm ${ }^{-1}$ : 2932, 1676, 1222, 970; ESI-HRMS calcd. for $\mathrm{C}_{25} \mathrm{H}_{25} \mathrm{FO}_{4}^{+}$ $\left([\mathrm{M}+\mathrm{H}]^{+}\right): 408.1737$, found: 408.1730 .

\section{4'-Fluoro-5,7-diisoprenyloxyflavone (4d)}

Yield: $73.3 \%$; mp 97-99 ${ }^{\circ} \mathrm{C}$; ${ }^{1} \mathrm{H}-\mathrm{NMR}$ (DMSO- $d_{6}$, $300 \mathrm{MHz}): \delta 1.66\left(\mathrm{~s}, 3 \mathrm{H},-\mathrm{CH}_{3}\right), 1.69\left(\mathrm{~s}, 3 \mathrm{H},-\mathrm{CH}_{3}\right), 1.70$ $\left(\mathrm{s}, 3 \mathrm{H},-\mathrm{CH}_{3}\right), 1.72\left(\mathrm{~s}, 3 \mathrm{H},-\mathrm{CH}_{3}\right), 4.63\left(\mathrm{~d}, 2 \mathrm{H},-\mathrm{CH}_{2}\right)$, $4.66\left(\mathrm{~d}, 2 \mathrm{H},-\mathrm{CH}_{2}\right), 5.43(\mathrm{t}, 1 \mathrm{H},=\mathrm{CH}), 5.45(\mathrm{t}, 1 \mathrm{H},=\mathrm{CH})$, 6.03-6.12 (m, 2H, $\left.-\mathrm{C}_{6} \mathrm{H}_{2}\right), 6.73(\mathrm{~s}, 1 \mathrm{H},=\mathrm{CH}), 6.90-7.29$ $\left(\mathrm{m}, 4 \mathrm{H},-\mathrm{C}_{6} \mathrm{H}_{4}\right) ;{ }^{13} \mathrm{C}-\mathrm{NMR}$ (DMSO- $d_{6}, 75 \mathrm{MHz}$ ): 19.2, 19.4, 25.2, 25.4, 45.1, 45.3, 96.8, 97.9, 103.5, 104.7, 115.2, $115.7,123.3,123.5,126.5,128.3,128.5,159.4,162.2$, 
162.5, 163.5, 168.4, 182.4; IR (KBr) cm $\mathrm{cm}^{-1}: 2932,1672$, 1221, 971; ESI-HRMS calcd. for $\mathrm{C}_{25} \mathrm{H}_{25} \mathrm{FO}_{4}^{+}\left([\mathrm{M}+\mathrm{H}]^{+}\right)$: 408.1737, found: 408.1746.

\section{2'-Chloro-5,7-diisoprenyloxyflavone (4e)}

Yield: $74.1 \%, \mathrm{mp} 105-107{ }^{\circ} \mathrm{C} ;{ }^{1} \mathrm{H}-\mathrm{NMR}$ (DMSO- $d_{6}$, $300 \mathrm{MHz}): \delta 1.67\left(\mathrm{~s}, 3 \mathrm{H},-\mathrm{CH}_{3}\right), 1.70\left(\mathrm{~s}, 3 \mathrm{H},-\mathrm{CH}_{3}\right), 1.71$ $\left(\mathrm{s}, 3 \mathrm{H},-\mathrm{CH}_{3}\right), 1.73\left(\mathrm{~s}, 3 \mathrm{H},-\mathrm{CH}_{3}\right), 4.58\left(\mathrm{~d}, 2 \mathrm{H},-\mathrm{CH}_{2}\right)$, $4.61\left(\mathrm{~d}, 2 \mathrm{H},-\mathrm{CH}_{2}\right), 5.42(\mathrm{t}, 1 \mathrm{H},=\mathrm{CH}), 5.47(\mathrm{t}, 1 \mathrm{H},=\mathrm{CH})$, 6.03-6.10 (m, 2H, $\left.-\mathrm{C}_{6} \mathrm{H}_{2}\right), 6.65(\mathrm{~s}, 1 \mathrm{H},=\mathrm{CH}), 7.06-7.28$ $\left(\mathrm{m}, 4 \mathrm{H},-\mathrm{C}_{6} \mathrm{H}_{4}\right) ;{ }^{13} \mathrm{C}-\mathrm{NMR}$ (DMSO- $\left.d_{6}, 75 \mathrm{MHz}\right): \delta 19.3$, 19.6, 25.3, 25.6, 45.3, 45.5, 97.5, 98.6, 103.6, 104.5, 123.5, $123.8,126.6,127.4,128.7,129.5,131.2,131.9,132.4$, 132.6, 159.2, 162.6, 163.7, 168.5, 182.5; IR $(\mathrm{KBr}) \mathrm{cm}^{-1}$ : 2943, 1676, 1220, 973; ESI-HRMS calcd. for $\mathrm{C}_{25} \mathrm{H}_{25} \mathrm{ClO}_{4}^{+}$ $\left([\mathrm{M}+\mathrm{H}]^{+}\right): 425.1441$, found: 425.1430 .

\section{3'-Chloro-5,7-diisoprenyloxyflavone (4f)}

Yield: 77\%; mp 119-121 ${ }^{\circ} \mathrm{C}$; ${ }^{1} \mathrm{H}-\mathrm{NMR}$ (DMSO- $d_{6}$, $300 \mathrm{MHz}): \delta 1.69\left(\mathrm{~s}, 3 \mathrm{H},-\mathrm{CH}_{3}\right), 1.71\left(\mathrm{~s}, 3 \mathrm{H},-\mathrm{CH}_{3}\right), 1.74$ $\left(\mathrm{s}, 3 \mathrm{H},-\mathrm{CH}_{3}\right), 1.75\left(\mathrm{~s}, 3 \mathrm{H},-\mathrm{CH}_{3}\right), 4.50\left(\mathrm{~d}, 2 \mathrm{H},-\mathrm{CH}_{2}\right)$, $4.54\left(\mathrm{~d}, 2 \mathrm{H},-\mathrm{CH}_{2}\right), 5.40(\mathrm{t}, 1 \mathrm{H},=\mathrm{CH}), 5.44(\mathrm{t}, 1 \mathrm{H},=\mathrm{CH})$, 6.02-6.11 (m, 2H, $\left.-\mathrm{C}_{6} \mathrm{H}_{2}\right), 6.68(\mathrm{~s}, 1 \mathrm{H},=\mathrm{CH}), 7.14-7.34$ $\left(\mathrm{m}, 4 \mathrm{H},-\mathrm{C}_{6} \mathrm{H}_{4}\right) ;{ }^{13} \mathrm{C}-\mathrm{NMR}$ (DMSO- $\left.d_{6}, 75 \mathrm{MHz}\right): \delta 19.6$, 19.9, 25.4, 25.8, 65.5, 65.8, 98.9, 99.4, 103.3, 104.8, 123.7, $123.9,124.5,126.7,128.3,130.7,131.6,132.5,132.8$, 134.5, 160.2, 162.4, 163.6, 169.1, 182.9; IR (KBr) cm $\mathrm{cm}^{-1}$ : 2940, 1677, 1222, 970; ESI-HRMS calcd. for $\mathrm{C}_{25} \mathrm{H}_{25} \mathrm{ClO}_{4}^{+}$ $\left([\mathrm{M}+\mathrm{H}]^{+}\right): 425.1441$, found: 425.1451 .

\section{4'-Chloro-5,7-diisoprenyloxyflavone (4g)}

Yield: $81 \%$; mp $117-119{ }^{\circ} \mathrm{C}$; ${ }^{1} \mathrm{H}-\mathrm{NMR}$ (DMSO- $d_{6}$, $300 \mathrm{MHz}): \delta 1.65\left(\mathrm{~s}, 3 \mathrm{H},-\mathrm{CH}_{3}\right), 1.67\left(\mathrm{~s}, 3 \mathrm{H},-\mathrm{CH}_{3}\right), 1.72(\mathrm{~s}$, $\left.3 \mathrm{H},-\mathrm{CH}_{3}\right), 1.74\left(\mathrm{~s}, 3 \mathrm{H},-\mathrm{CH}_{3}\right), 4.62\left(\mathrm{~d}, 2 \mathrm{H},-\mathrm{CH}_{2}\right), 4.64(\mathrm{~d}$, $\left.2 \mathrm{H},-\mathrm{CH}_{2}\right), 5.40(\mathrm{t}, 1 \mathrm{H},=\mathrm{CH}), 5.43(\mathrm{t}, 1 \mathrm{H},=\mathrm{CH}), 6.05-6.12$ $\left(\mathrm{m}, 2 \mathrm{H},-\mathrm{C}_{6} \mathrm{H}_{2}\right), 6.81(\mathrm{~s}, 1 \mathrm{H},=\mathrm{CH}), 7.21-7.26(\mathrm{~m}, 4 \mathrm{H}$, $-\mathrm{C}_{6} \mathrm{H}_{4}$ ); ${ }^{13} \mathrm{C}-\mathrm{NMR}$ (DMSO- $d_{6}, 75 \mathrm{MHz}$ ): $\delta 20.1,20.3$, 25.4, 25.7, 65.6, 65.8, 99.2, 100.1, 103.8, 104.7, 123.7, $124.0,127.3,127.8,128.2,128.5,129.2,132.2,132.5$, 133.7, 159.6, 162.4, 163.5, 168.7, 182.9; IR (KBr) cm cm$^{-1}$ : 2943, 1676, 1220, 973; ESI-HRMS calcd. for $\mathrm{C}_{25} \mathrm{H}_{25} \mathrm{ClO}_{4}^{+}$ $\left([\mathrm{M}+\mathrm{H}]^{+}\right):$425.1441, found: 425.1449 .

\section{2'-Bromo-5,7-diisoprenyloxyflavone (4h)}

Yield: 69\%; mp 120-122 ${ }^{\circ} \mathrm{C} ;{ }^{1} \mathrm{H}-\mathrm{NMR}$ (DMSO- $d_{6}$, $300 \mathrm{MHz}): \delta 1.68\left(\mathrm{~s}, 3 \mathrm{H},-\mathrm{CH}_{3}\right), 1.70\left(\mathrm{~s}, 3 \mathrm{H},-\mathrm{CH}_{3}\right), 1.73$ $\left(\mathrm{s}, 3 \mathrm{H},-\mathrm{CH}_{3}\right), 1.75\left(\mathrm{~s}, 3 \mathrm{H},-\mathrm{CH}_{3}\right), 4.48\left(\mathrm{~d}, 2 \mathrm{H},-\mathrm{CH}_{2}\right)$, $4.50\left(\mathrm{~d}, 2 \mathrm{H},-\mathrm{CH}_{2}\right), 5.42(\mathrm{t}, 1 \mathrm{H},=\mathrm{CH}), 5.47(\mathrm{t}, 1 \mathrm{H},=\mathrm{CH})$, 6.05-6.13 (m, 2H, $\left.-\mathrm{C}_{6} \mathrm{H}_{2}\right), 6.77(\mathrm{~s}, 1 \mathrm{H},=\mathrm{CH}), 7.06-7.41$ $\left(\mathrm{m}, 4 \mathrm{H},-\mathrm{C}_{6} \mathrm{H}_{4}\right) ;{ }^{13} \mathrm{C}-\mathrm{NMR}$ (DMSO- $d_{6}, 75 \mathrm{MHz}$ ): $\delta 19.2$, 19.7, 25.4, 25.6, 65.3, 65.5, 98.4, 99.2, 103.1, 104.4, 118.3,
123.6, 123.9, 127.1, 128.6, 130.4, 131.6, 132.3, 132.5, 138.4, 159.4, 162.7, 164.1, 168.5, 182.6; IR (KBr) cm ${ }^{-1}$ : 2936, 1674, 1220, 968; ESI-HRMS calcd. for $\mathrm{C}_{25} \mathrm{H}_{25} \mathrm{BrO}_{4}^{+}$ $\left([\mathrm{M}+\mathrm{H}]^{+}\right): 469.0936$, found: 469.0915 .

\section{3'-Bromo-5,7-diisoprenyloxyflavone (4i)}

Yield: 70\%; mp 114-117 ${ }^{\circ} \mathrm{C} ;{ }^{1} \mathrm{H}-\mathrm{NMR}$ (DMSO- $d_{6}$, $300 \mathrm{MHz}): \delta 1.67\left(\mathrm{~s}, 3 \mathrm{H},-\mathrm{CH}_{3}\right), 1.69\left(\mathrm{~s}, 3 \mathrm{H},-\mathrm{CH}_{3}\right), 1.70$ $\left(\mathrm{s}, 3 \mathrm{H},-\mathrm{CH}_{3}\right), 1.72\left(\mathrm{~s}, 3 \mathrm{H},-\mathrm{CH}_{3}\right), 4.56\left(\mathrm{~d}, 2 \mathrm{H},-\mathrm{CH}_{2}\right)$, $4.59\left(\mathrm{~d}, 2 \mathrm{H},-\mathrm{CH}_{2}\right), 5.40(\mathrm{t}, 1 \mathrm{H},=\mathrm{CH}), 5.43(\mathrm{t}, 1 \mathrm{H},=\mathrm{CH})$, 6.03-6.11 (m, 2H, $\left.-\mathrm{C}_{6} \mathrm{H}_{2}\right), 6.79(\mathrm{~s}, 1 \mathrm{H},=\mathrm{CH}), 7.12-7.50$ (m, $\left.4 \mathrm{H},-\mathrm{C}_{6} \mathrm{H}_{4}\right) ;{ }^{13} \mathrm{C}-\mathrm{NMR}$ (DMSO- $\left.d_{6}, 75 \mathrm{MHz}\right): \delta 19.3$, 19.5, 25.5, 25.7, 64.8, 65.0, 96.4, 97.7, 103.3, 104.5, 122.8, $123.8,124.0,125.7,129.1,130.2,130.5,131.9,132.3$, 132.5, 162.4, 163.3, 163.9, 168.5, 182.6; IR (KBr) cm ${ }^{-1}$ : 2941, 1677, 1222, 972; ESI-HRMS calcd. for $\mathrm{C}_{25} \mathrm{H}_{26} \mathrm{O}_{4}^{+}$ $\left([\mathrm{M}+\mathrm{H}]^{+}\right)$: 469.0936, found: 469.0943 .

\section{4'-Bromo-5,7-diisoprenyloxyflavone (4j)}

Yield: $78.5 \%, \mathrm{mp} 122-124{ }^{\circ} \mathrm{C} ;{ }^{1} \mathrm{H}-\mathrm{NMR}$ (DMSO- $d_{6}$, $300 \mathrm{MHz}): \delta 1.65\left(\mathrm{~s}, 3 \mathrm{H},-\mathrm{CH}_{3}\right), 1.68\left(\mathrm{~s}, 3 \mathrm{H},-\mathrm{CH}_{3}\right), 1.71$ $\left(\mathrm{s}, 3 \mathrm{H},-\mathrm{CH}_{3}\right), 1.73\left(\mathrm{~s}, 3 \mathrm{H},-\mathrm{CH}_{3}\right), 4.62\left(\mathrm{~d}, 2 \mathrm{H},-\mathrm{CH}_{2}\right)$, $4.65\left(\mathrm{~d}, 2 \mathrm{H},-\mathrm{CH}_{2}\right), 5.39(\mathrm{t}, 1 \mathrm{H},=\mathrm{CH}), 5.42(\mathrm{t}, 1 \mathrm{H},=\mathrm{CH})$, 6.04-6.13 (m, 2H, $\left.-\mathrm{C}_{6} \mathrm{H}_{2}\right), 6.76(\mathrm{~s}, 1 \mathrm{H},=\mathrm{CH}), 7.21-7.42$ $\left(\mathrm{m}, 4 \mathrm{H},-\mathrm{C}_{6} \mathrm{H}_{4}\right) ;{ }^{13} \mathrm{C}-\mathrm{NMR}$ (DMSO- $\left.d_{6}, 75 \mathrm{MHz}\right): \delta 19.6$, 19.8, 25.4, 25.7, 66.1, 66.5, 96.8, 97.9, 103.7, 104.6, 122.4, 123.4, 123.7, 128.1, 128.4, 129.9, 131.3, 131.4, 132.3, 132.5, 160.0, 162.7, 163.8, 168.6, 182.5; IR (KBr) cm $\mathrm{cm}^{-1}$ : 2941, 1678, 1222, 972; ESI-HRMS calcd. for $\mathrm{C}_{25} \mathrm{H}_{26} \mathrm{O}_{4}^{+}$ $\left([\mathrm{M}+\mathrm{H}]^{+}\right): 469.0936$, found: 469.0921 .

\section{2',4'-Dichloro-5,7-diisoprenyloxyflavone (4k)}

Yield: $78.4 \%$, mp $107-108{ }^{\circ} \mathrm{C}$; ${ }^{1} \mathrm{H}-\mathrm{NMR}$ (DMSO- $d_{6}$, $300 \mathrm{MHz}): \delta 1.67\left(\mathrm{~s}, 3 \mathrm{H},-\mathrm{CH}_{3}\right), 1.69\left(\mathrm{~s}, 3 \mathrm{H},-\mathrm{CH}_{3}\right), 1.73(\mathrm{~s}$, $\left.3 \mathrm{H},-\mathrm{CH}_{3}\right), 1.75\left(\mathrm{~s}, 3 \mathrm{H},-\mathrm{CH}_{3}\right), 4.48\left(\mathrm{~d}, 2 \mathrm{H},-\mathrm{CH}_{2}\right), 4.50(\mathrm{~d}$, $\left.2 \mathrm{H},-\mathrm{CH}_{2}\right), 5.42(\mathrm{t}, 1 \mathrm{H},=\mathrm{CH}), 5.45(\mathrm{t}, 1 \mathrm{H},=\mathrm{CH}), 6.05-6.11$ $\left(\mathrm{m}, 2 \mathrm{H},-\mathrm{C}_{6} \mathrm{H}_{2}\right), 6.78(\mathrm{~s}, 1 \mathrm{H},=\mathrm{CH}), 7.10-7.28(\mathrm{~m}, 3 \mathrm{H}$, $\left.-\mathrm{C}_{6} \mathrm{H}_{3}\right) ;{ }^{13} \mathrm{C}-\mathrm{NMR}$ (DMSO- $d_{6}, 75 \mathrm{MHz}$ ): $\delta 19.6,19.8,25.5$, $25.8,65.5,65.7,96.4,97.8,103.7,104.5,123.5,123.8$, $127.1,129.3,129.6,130.4,132.4,132.7,132.9,159.7$, 162.3, 163.8, 168.5, 182.5; IR (KBr) cm $\mathrm{cm}^{-1}: 2941,1676$, 1221, 968; ESI-HRMS calcd. for $\mathrm{C}_{25} \mathrm{H}_{24} \mathrm{Cl}_{2} \mathrm{O}_{4}^{+}\left([\mathrm{M}+\mathrm{H}]^{+}\right)$: 459.1052, found: 459.1041.

\section{4'-Nitro-5,7-diisoprenyloxyflavone (4I)}

Yield: $60 \%$, mp $91-93{ }^{\circ} \mathrm{C} ;{ }^{1} \mathrm{H}-\mathrm{NMR}$ (DMSO- $d_{6}, 300$ $\mathrm{MHz}): \delta 1.69\left(\mathrm{~s}, 3 \mathrm{H},-\mathrm{CH}_{3}\right), 1.70\left(\mathrm{~s}, 3 \mathrm{H},-\mathrm{CH}_{3}\right), 1.73(\mathrm{~s}, 3 \mathrm{H}$, $\left.-\mathrm{CH}_{3}\right), 1.76\left(\mathrm{~s}, 3 \mathrm{H},-\mathrm{CH}_{3}\right), 4.62\left(\mathrm{~d}, 2 \mathrm{H},-\mathrm{CH}_{2}\right), 4.67(\mathrm{~d}, 2 \mathrm{H}$, $\left.-\mathrm{CH}_{2}\right), 5.39(\mathrm{t}, 1 \mathrm{H},=\mathrm{CH}), 5.43(\mathrm{t}, 1 \mathrm{H},=\mathrm{CH}), 6.05-6.11(\mathrm{~m}$, $\left.2 \mathrm{H},-\mathrm{C}_{6} \mathrm{H}_{2}\right), 7.02(\mathrm{~s}, 1 \mathrm{H},=\mathrm{CH}), 7.76-8.43\left(\mathrm{~m}, 4 \mathrm{H},-\mathrm{C}_{6} \mathrm{H}_{4}\right)$; ${ }^{13} \mathrm{C}-\mathrm{NMR}\left(\mathrm{DMSO}-d_{6}, 75 \mathrm{MHz}\right): \delta 19.5,19.7,25.5,25.7$, 
65.5, 65.8, 98.7, 99.9, 103.8, 104.3, 121.3, 121.5, 123.5, $123.7,127.6,127.9,132.4,132.7,136.5,148.8,160.2$ 162.5, 163.7, 168.4, 182.5; IR (KBr) cm $\mathrm{cm}^{-1}: 2941,1676$, 1221, 972; ESI-HRMS calcd. for $\mathrm{C}_{25} \mathrm{H}_{25} \mathrm{NO}_{6}{ }^{+}\left([\mathrm{M}+\mathrm{H}]^{+}\right)$: 436.1682, found: 436.1669 .

\section{2'-Methyl-5,7-diisoprenyloxyflavone (4m)}

Yield: 74.4\%, mp 96-98 ${ }^{\circ} \mathrm{C}$; ${ }^{1} \mathrm{H}-\mathrm{NMR}$ (DMSO- $d_{6}$, $300 \mathrm{MHz}): \delta 1.69\left(\mathrm{~s}, 3 \mathrm{H},-\mathrm{CH}_{3}\right), 1.70\left(\mathrm{~s}, 3 \mathrm{H},-\mathrm{CH}_{3}\right), 1.71$ $\left(\mathrm{s}, 3 \mathrm{H},-\mathrm{CH}_{3}\right), 1.73\left(\mathrm{~s}, 3 \mathrm{H},-\mathrm{CH}_{3}\right), 2.40\left(\mathrm{~s}, 3 \mathrm{H},-\mathrm{CH}_{3}\right), 4.45$ $\left(\mathrm{d}, 2 \mathrm{H},-\mathrm{CH}_{2}\right), 4.47\left(\mathrm{~d}, 2 \mathrm{H},-\mathrm{CH}_{2}\right), 5.41(\mathrm{t}, 1 \mathrm{H},=\mathrm{CH})$, $5.43(\mathrm{t}, 1 \mathrm{H},=\mathrm{CH}), 6.03-6.12\left(\mathrm{~m}, 2 \mathrm{H},-\mathrm{C}_{6} \mathrm{H}_{2}\right), 6.56(\mathrm{~s}, 1 \mathrm{H}$, $=\mathrm{CH}), 7.03-7.21\left(\mathrm{~m}, 4 \mathrm{H},-\mathrm{C}_{6} \mathrm{H}_{4}\right){ }^{13} \mathrm{C}-\mathrm{NMR}\left(\mathrm{DMSO}-d_{6}, 75\right.$ MHz): $\delta 18.4,19.5,19.7,25.3,25.6,65.5,65.7,97.5,98.7$, $103.5,104.6,123.3,123.5,125.7,126.5,127.3,128.7$, 129.3, 132.2, 132.5, 159.8, 162.7, 163.8, 168.5, 182.6; IR $(\mathrm{KBr}) \mathrm{cm}^{-1}: 2939,1676,1221,971$; ESI-HRMS calcd. for $\mathrm{C}_{26} \mathrm{H}_{28} \mathrm{O}_{4}^{+}\left([\mathrm{M}+\mathrm{H}]^{+}\right): 405.1988$, found: 405.1968 .

\section{3'-Methyl-5,7-diisoprenyloxyflavone (4n)}

Yield: $76 \%$, mp $101-104{ }^{\circ} \mathrm{C} ;{ }^{1} \mathrm{H}-\mathrm{NMR}$ (DMSO- $d_{6}$, $300 \mathrm{MHz}): \delta 1.69\left(\mathrm{~s}, 3 \mathrm{H},-\mathrm{CH}_{3}\right), 1.71\left(\mathrm{~s}, 3 \mathrm{H},-\mathrm{CH}_{3}\right), 1.72$ $\left(\mathrm{s}, 3 \mathrm{H},-\mathrm{CH}_{3}\right), 1.75\left(\mathrm{~s}, 3 \mathrm{H},-\mathrm{CH}_{3}\right), 2.39\left(\mathrm{~s}, 3 \mathrm{H},-\mathrm{CH}_{3}\right), 4.42$ $\left(\mathrm{d}, 2 \mathrm{H},-\mathrm{CH}_{2}\right), 4.44\left(\mathrm{~d}, 2 \mathrm{H},-\mathrm{CH}_{2}\right), 5.40(\mathrm{t}, 1 \mathrm{H},=\mathrm{CH}), 5.42$ $(\mathrm{t}, 1 \mathrm{H},=\mathrm{CH}), 6.03-6.12\left(\mathrm{~m}, 2 \mathrm{H},-\mathrm{C}_{6} \mathrm{H}_{2}\right), 6.73(\mathrm{~s}, 1 \mathrm{H},=\mathrm{CH})$, 6.95-7.13 (m, 4H, $\left.-\mathrm{C}_{6} \mathrm{H}_{4}\right) ;{ }^{13} \mathrm{C}-\mathrm{NMR}$ (DMSO- $d_{6}, 75 \mathrm{MHz}$ ): $\delta 19.8,20.1,24.3,25.5,25.8,65.6,65.9,98.9,100.2$, $103.3,104.5,123.3,123.7,123.9,126.2,128.3,128.8$, $130.3,132.5,132.7,138.7,159.7,162.5,163.6,168.8$, 182.4; IR (KBr) cm ${ }^{-1}: 2942,1677,1222,970$; ESI-HRMS calcd. for $\mathrm{C}_{26} \mathrm{H}_{28} \mathrm{O}_{4}^{+}\left([\mathrm{M}+\mathrm{H}]^{+}\right)$: 405.1988, found: 405.1971 .

\section{4'-Methyl-5,7-diisoprenyloxyflavone (4o)}

Yield: $85.6 \%, \mathrm{mp} 113-115{ }^{\circ} \mathrm{C}$; ${ }^{1} \mathrm{H}-\mathrm{NMR}$ (DMSO- $d_{6}$, $300 \mathrm{MHz}): \delta 1.67\left(\mathrm{~s}, 3 \mathrm{H},-\mathrm{CH}_{3}\right), 1.71\left(\mathrm{~s}, 3 \mathrm{H},-\mathrm{CH}_{3}\right), 1.73(\mathrm{~s}$, $\left.3 \mathrm{H},-\mathrm{CH}_{3}\right), 1.74\left(\mathrm{~s}, 3 \mathrm{H},-\mathrm{CH}_{3}\right), 2.38\left(\mathrm{~s}, 3 \mathrm{H},-\mathrm{CH}_{3}\right), 4.51(\mathrm{~d}$, $\left.2 \mathrm{H},-\mathrm{CH}_{2}\right), 4.53\left(\mathrm{~d}, 2 \mathrm{H},-\mathrm{CH}_{2}\right), 5.40(\mathrm{t}, 1 \mathrm{H},=\mathrm{CH}), 5.42(\mathrm{t}$, $1 \mathrm{H},=\mathrm{CH}), 6.05-6.13\left(\mathrm{~m}, 2 \mathrm{H},-\mathrm{C}_{6} \mathrm{H}_{2}\right), 6.75(\mathrm{~s}, 1 \mathrm{H},=\mathrm{CH})$, 7.01-7.19 (m, 4H, $\left.-\mathrm{C}_{6} \mathrm{H}_{4}\right) ;{ }^{13} \mathrm{C}-\mathrm{NMR}$ (DMSO- $d_{6}, 75 \mathrm{MHz}$ ): $\delta$ 19.7, 19.9, 24.1, 25.5, 25.8, 65.6, 65.9, 98.8, 99.6, 103.4, $104.5,123.6,123.8,126.2,126.4,127.5,129.2,129.3$, 132.4, 132.7, 137.6, 159.9, 162.8, 163.5, 168.6, 182.4; IR $(\mathrm{KBr}) \mathrm{cm}^{-1}: 2941,1676,1220,970$; ESI-HRMS calcd. for $\mathrm{C}_{26} \mathrm{H}_{28} \mathrm{O}_{4}^{+}\left([\mathrm{M}+\mathrm{H}]^{+}\right): 405.1988$, found: 405.1990 .

\section{2'-Methoxy-5,7-diisoprenyloxyflavone (4p)}

Yield: $68.4 \%, \mathrm{mp} 80-83{ }^{\circ} \mathrm{C}$; ${ }^{1} \mathrm{H}-\mathrm{NMR}$ (DMSO- $d_{6}$, $300 \mathrm{MHz}): \delta 1.68\left(\mathrm{~s}, 3 \mathrm{H},-\mathrm{CH}_{3}\right), 1.70\left(\mathrm{~s}, 3 \mathrm{H},-\mathrm{CH}_{3}\right), 1.72$ $\left(\mathrm{s}, 3 \mathrm{H},-\mathrm{CH}_{3}\right), 1.74\left(\mathrm{~s}, 3 \mathrm{H},-\mathrm{CH}_{3}\right), 3.74\left(\mathrm{~s}, 3 \mathrm{H},-\mathrm{O} \mathrm{CH}_{3}\right)$, $4.63\left(\mathrm{~d}, 2 \mathrm{H},-\mathrm{CH}_{2}\right), 4.65\left(\mathrm{~d}, 2 \mathrm{H},-\mathrm{CH}_{2}\right), 5.41(\mathrm{t}, 1 \mathrm{H},=\mathrm{CH})$, $5.44(\mathrm{t}, 1 \mathrm{H},=\mathrm{CH}), 6.04-6.11\left(\mathrm{~m}, 2 \mathrm{H},-\mathrm{C}_{6} \mathrm{H}_{2}\right), 6.73(\mathrm{~s}, 1 \mathrm{H}$,
$=\mathrm{CH}), 6.73-7.20\left(\mathrm{~m}, 4 \mathrm{H},-\mathrm{C}_{6} \mathrm{H}_{4}\right) ;{ }^{13} \mathrm{C}-\mathrm{NMR}$ (DMSO- $d_{6}, 75$ MHz): $\delta 19.7,20.0,25.6,25.8,56.5,65.1,65.4,96.7,98.9$, 103.3, 104.6, 110.7, 114.4, 121.3, 123.4, 123.6, 127.2, 129.6, 132.6, 132.7, 159.7, 162.0, 163.4, 168.4, 182.6; IR $(\mathrm{KBr}) \mathrm{cm}^{-1}: 2943,1678,1221,970$; ESI-HRMS calcd. for $\mathrm{C}_{26} \mathrm{H}_{28} \mathrm{O}_{5}^{+}\left([\mathrm{M}+\mathrm{H}]^{+}\right): 421.1937$, found: 421.1921 .

\section{3'-Methoxy-5,7-diisoprenyloxyflavone (4q)}

Yield: $65.2 \%$, mp. $77-79{ }^{\circ} \mathrm{C}$; ${ }^{1} \mathrm{H}-\mathrm{NMR}$ (DMSO- $d_{6}$, $300 \mathrm{MHz}): \delta 1.66\left(\mathrm{~s}, 3 \mathrm{H},-\mathrm{CH}_{3}\right), 1.67\left(\mathrm{~s}, 3 \mathrm{H},-\mathrm{CH}_{3}\right), 1.71$ $\left(\mathrm{s}, 3 \mathrm{H},-\mathrm{CH}_{3}\right), 1.73\left(\mathrm{~s}, 3 \mathrm{H},-\mathrm{CH}_{3}\right), 3.71\left(\mathrm{~s}, 3 \mathrm{H},-\mathrm{O} \mathrm{CH}_{3}\right)$, $4.45\left(\mathrm{~d}, 2 \mathrm{H},-\mathrm{CH}_{2}\right), 4.48\left(\mathrm{~d}, 2 \mathrm{H},-\mathrm{CH}_{2}\right), 5.39(\mathrm{t}, 1 \mathrm{H},=\mathrm{CH})$, $5.43(\mathrm{t}, 1 \mathrm{H},=\mathrm{CH}), 6.06-6.12\left(\mathrm{~m}, 2 \mathrm{H},-\mathrm{C}_{6} \mathrm{H}_{2}\right), 6.59(\mathrm{~s}, 1 \mathrm{H}$, $=\mathrm{CH}), 6.71-7.19\left(\mathrm{~m}, 4 \mathrm{H},-\mathrm{C}_{6} \mathrm{H}_{4}\right) ;{ }^{13} \mathrm{C}-\mathrm{NMR}\left(\mathrm{DMSO}-d_{6}\right.$, $75 \mathrm{MHz}): \delta 19.8,20.1,25.6,25.8,56.0,64.9,65.4,98.8$, $100.1,103.2,104.3,110.3113 .5,118.5,123.5,123.6$, $129.8,131.4,132.5,132.8,159.8,162.5,163.8,168.3$, 182.6; IR (KBr) cm ${ }^{-1}: 2942,1677,1220,971$; ESI-HRMS calcd. for $\mathrm{C}_{26} \mathrm{H}_{28} \mathrm{O}_{5}^{+}\left([\mathrm{M}+\mathrm{H}]^{+}\right): 421.1937$, found: 421.1921 .

\section{4'-Methoxy-5,7-diisoprenyloxyflavone (4r)}

Yield: $81.1 \%, \mathrm{mp} 88-90{ }^{\circ} \mathrm{C} ;{ }^{1} \mathrm{H}-\mathrm{NMR}$ (DMSO- $d_{6}$, $300 \mathrm{MHz}): \delta 1.69\left(\mathrm{~s}, 3 \mathrm{H},-\mathrm{CH}_{3}\right), 1.70\left(\mathrm{~s}, 3 \mathrm{H},-\mathrm{CH}_{3}\right), 1.71$ $\left(\mathrm{s}, 3 \mathrm{H},-\mathrm{CH}_{3}\right), 1.72\left(\mathrm{~s}, 3 \mathrm{H},-\mathrm{CH}_{3}\right), 3.71(\mathrm{~s}, 3 \mathrm{H},-\mathrm{O} \mathrm{CH})$, $4.50\left(\mathrm{~d}, 2 \mathrm{H},-\mathrm{CH}_{2}\right), 4.52\left(\mathrm{~d}, 2 \mathrm{H},-\mathrm{CH}_{2}\right), 5.39(\mathrm{t}, 1 \mathrm{H},=\mathrm{CH})$, $5.42(\mathrm{t}, 1 \mathrm{H},=\mathrm{CH}), 6.02-6.10\left(\mathrm{~m}, 2 \mathrm{H},-\mathrm{C}_{6} \mathrm{H}_{2}\right), 6.69(\mathrm{~s}, 1 \mathrm{H}$, $=\mathrm{CH}), 6.67-7.13\left(\mathrm{~m}, 4 \mathrm{H},-\mathrm{C}_{6} \mathrm{H}_{4}\right),{ }^{13} \mathrm{C}-\mathrm{NMR}\left(\mathrm{DMSO}-d_{6}, 75\right.$ MHz): $\delta 19.4,20.3,25.4,25.6,55.9,65.8,60.2,97.8,99.9$, $103.4,104.5,114.7,114.9,122.7,123.3,123.5,127.4$, 127.6, 132.4, 132.7, 160.1, 162.3, 163.6, 168.5, 182.4; IR $(\mathrm{KBr}) \mathrm{cm}^{-1}: 2941,1675,1221,969$; ESI-HRMS calcd. for $\mathrm{C}_{26} \mathrm{H}_{28} \mathrm{O}_{5}^{+}\left([\mathrm{M}+\mathrm{H}]^{+}\right): 421.1937$, found: 421.1942.

\section{4'-Dimethylamine-5,7-diisoprenyloxyflavone (4s)}

Yield: $63 \%, \mathrm{mp} 83-85{ }^{\circ} \mathrm{C} ;{ }^{1} \mathrm{H}-\mathrm{NMR}$ (DMSO- $d_{6}$, $300 \mathrm{MHz}): \delta 1.68\left(\mathrm{~s}, 3 \mathrm{H},-\mathrm{CH}_{3}\right), 1.69\left(\mathrm{~s}, 3 \mathrm{H},-\mathrm{CH}_{3}\right)$, $1.70\left(\mathrm{~s}, 3 \mathrm{H},-\mathrm{CH}_{3}\right), 1.73\left(\mathrm{~s}, 3 \mathrm{H},-\mathrm{CH}_{3}\right), 2.80,2.89(\mathrm{~s}, 6 \mathrm{H}$, $\left.-\mathrm{N}\left(\mathrm{CH}_{3}\right)_{2}\right), 4.61\left(\mathrm{~d}, 2 \mathrm{H},-\mathrm{CH}_{2}\right), 4.63\left(\mathrm{~d}, 2 \mathrm{H},-\mathrm{CH}_{2}\right), 5.40$ (t, $1 \mathrm{H},=\mathrm{CH}), 5.42(\mathrm{t}, 1 \mathrm{H},=\mathrm{CH}), 6.03-6.11\left(\mathrm{~m}, 2 \mathrm{H},-\mathrm{C}_{6} \mathrm{H}_{2}\right)$, $6.76(\mathrm{~s}, 1 \mathrm{H},=\mathrm{CH}), 6.60-7.16\left(\mathrm{~m}, 4 \mathrm{H},-\mathrm{C}_{6} \mathrm{H}_{4}\right) ;{ }^{13} \mathrm{C}-\mathrm{NMR}$ (DMSO- $\left.d_{6}, 75 \mathrm{MHz}\right): \delta 19.8,20.2,25.4,25.7,40.5,40.8$, 65.4, 65.6, 97.8, 99.6, 103.4, 104.5, 114.3, 114.5, 119.3, $123.3,123.4,127.1,127.4,132.4,132.5,148.4,160.0$, 162.5, 163.4, 168.4, 182.5; IR (KBr) cm $\mathrm{cm}^{-1}: 2940,1676$, 1220, 970; ESI-HRMS calcd. for $\mathrm{C}_{27} \mathrm{H}_{31} \mathrm{NO}_{4}{ }^{+}\left([\mathrm{M}+\mathrm{H}]^{+}\right)$: 434.2253, found: 434.2243 .

\section{3-Methoxy-4'-hydroxy-5,7-diisoprenyloxyflavone (4t)}

Yield: $73.4 \%$; obtained as an oil; ${ }^{1} \mathrm{H}-\mathrm{NMR}$ (DMSO- $\left.d_{6}, 300 \mathrm{MHz}\right): \delta 1.67\left(\mathrm{~s}, 3 \mathrm{H},-\mathrm{CH}_{3}\right), 1.69(\mathrm{~s}, 3 \mathrm{H}$, $\left.-\mathrm{CH}_{3}\right), 1.71\left(\mathrm{~s}, 3 \mathrm{H},-\mathrm{CH}_{3}\right), 1.73\left(\mathrm{~s}, 3 \mathrm{H},-\mathrm{CH}_{3}\right), 3.75(\mathrm{~s}, 3 \mathrm{H}$, 
$\left.-\mathrm{OCH}_{3}\right), 4.60\left(\mathrm{~d}, 2 \mathrm{H},-\mathrm{CH}_{2}\right), 4.63\left(\mathrm{~d}, 2 \mathrm{H},-\mathrm{CH}_{2}\right), 5.41(\mathrm{t}$, $1 \mathrm{H},=\mathrm{CH}), 5.44(\mathrm{t}, 1 \mathrm{H},=\mathrm{CH}), 6.03-6.12\left(\mathrm{~m}, 2 \mathrm{H},-\mathrm{C}_{6} \mathrm{H}_{2}\right)$, $6.73(\mathrm{~s}, 1 \mathrm{H},=\mathrm{CH}), 6.53-6.79\left(\mathrm{~m}, 3 \mathrm{H},-\mathrm{C}_{6} \mathrm{H}_{3}\right), 9.12(\mathrm{~s}$, $1 \mathrm{H},-\mathrm{OH}) ;{ }^{13} \mathrm{C}-\mathrm{NMR}$ (DMSO- $\left.d_{6}, 75 \mathrm{MHz}\right): \delta 19.7,19.9$, $25.7,25.9,56.8,65.6,65.8,98.9,99.9,103.6,104.3$, 113.4, 115.4, 120.3, 123.4, 123.7, 124.6, 132.5, 132.7, $145.7,150.1,159.7,162.2,163.5,168.5,182.3$; IR (KBr) $\mathrm{cm}^{-1}: 3250,2942,1678,1221,970$; ESI-HRMS calcd. for $\mathrm{C}_{26} \mathrm{H}_{28} \mathrm{O}_{6}^{+}\left([\mathrm{M}+\mathrm{H}]^{+}\right): 437.1886$, found: 437.1864 .

\section{Evaluation of the antibacterial activity in vitro}

The microorganisms used in the present study were S. aureus (S. aureus KCTC 503, S. aureus RN4220, and S. aureus KCTC 209), S. mutans (S. mutans KCTC 3289 and $S$. mutans KCTC 3065), and Escherichia coli (E. coli 1924 and $E$. coli 1356). The strains of multidrug-resistant clinical isolates used were multidrug-resistant $S$. aureus (MRSA CCARM 3167 and MRSA CCARM 3506) and quinolone-resistant $S$. aureus (QRSA CCARM 3505 and QRSA CCARM 3519). Clinical isolates were collected from various patients hospitalized in several clinics (Hosseinkhani et al., 2016; Fan, Reichling, Wink, 2013; Sharma et al., 2011).

A twofold serial dilution technique (Song et al., 2013) was used to determine the minimum inhibitory concentrations (MICs) of the compounds against susceptible microorganisms in the preliminary test (Grampositive and Gram-negative bacteria) and against strains of clinical isolates of multidrug-resistant Gram-positive bacteria. The compounds dissolved in DMSO and two-fold diluted at concentrations from $200 \mu \mathrm{g} / \mathrm{mL}$ to $0.1 \mu \mathrm{g} / \mathrm{mL}$, and they were added to culture media (Brain heart infusion for S. mutans and Mueller-Hinton agar for other bacteria) to obtain final concentrations of $0.5-64 \mu \mathrm{g} / \mathrm{mL}$. The final amount of bacteria applied was $105 \mathrm{CFU} / \mathrm{mL}$. MIC values were determined after incubation at $37^{\circ} \mathrm{C}$ for $20 \mathrm{~h}$. The lowest concentration of the test substance that completely inhibited microorganism growth was recorded as the MIC (expressed in $\mu \mathrm{M}$ ). Norfloxacin was used as the drug standard. All experiments were conducted in triplicate.

\section{RESULTS AND DISCUSSION}

\section{Chemistry}

The target compounds were obtained as outlined in Scheme 1. Compounds 1a-1t were synthesized from the Claisen-Schmidt condensation of commercially available 2,4,6-trihydroxyacetophenone (protected as methoxymethyl ethers) with different substituted aromatic aldehydes in aqueous ethanolic sodium hydroxide (Zhen et al., 2016). Intermediates 1a-1t were then treated with $3 \mathrm{M} \mathrm{HCl}$ in methanol to yield 2,4,6-trihydroxychalcones derivatives $\mathbf{2 a - 2 t}$ (Xie et al., 2014). Subsequent substitution with prenyl bromide in acetone under reflux in the presence of anhydrous $\mathrm{K}_{2} \mathrm{CO}_{3}$ afforded compounds 3a-3t (Wang et al., 2015). 5,7-Diisoprenyloxyflavone derivatives $\mathbf{4 a - 4 t}$ were obtained in good yields by treating $\mathbf{3 a}-\mathbf{3 t}$ with $\mathrm{I}_{2}$ in DMSO. The chemical structures of the target compounds were characterized by IR, ${ }^{1} \mathrm{H}-\mathrm{NMR},{ }^{13} \mathrm{C}-\mathrm{NMR}$, and highresolution mass spectroscopy. The IR spectra of the newly synthesized 5,7-diisoprenyloxyflavone derivatives $\mathbf{4 a}-\mathbf{4 t}$ showed absorption stretching bands at 2931-2943 $\mathrm{cm}^{-1}$ and $1672-1678 \mathrm{~cm}^{-1}$ stretching $\left(1220-1222 \mathrm{~cm}^{-1}\right)$ corresponding to $\left(-\mathrm{CH}_{3}\right),(\mathrm{C}=\mathrm{O})$ and $(\mathrm{C}-\mathrm{O}-\mathrm{C})$ group, respectively. IR spectrum of compound $4 \mathbf{t}$ showed absorption bands at $3250 \mathrm{~cm}^{-1}$ corresponding to stretching absorption of - $\mathrm{OH}$ group. The characteristic feature in the ${ }^{1} \mathrm{H}-\mathrm{NMR}$ spectrum of compound $\mathbf{2} \mathbf{t}$ is the appearance of two triplet peaks at 5.41 and $5.44 \mathrm{ppm}$ which represented the prenyl protons in $(\mathrm{CH}=\mathrm{C}-)$ group. Furthermore, two singlet peaks at $6.73 \mathrm{ppm}$ and $9.12 \mathrm{ppm}$ related to flavone ring $3-\mathrm{C}$ protons in $(\mathrm{CH}=)$ group and at phenyl ring $4^{\prime}-\mathrm{C}$ protons in $(-\mathrm{OH})$ group were observed. ${ }^{1} \mathrm{H}-\mathrm{NMR}$ spectra showed the $=\mathrm{CH}$ protons of the group at $6.56-7.02 \mathrm{ppm}$, The characteristic feature in the ${ }^{13} \mathrm{C}$-NMR spectrum of compound $2 \mathbf{t}$ displayed four signals peakes in $\left(-\mathrm{CH}_{3}\right)$ group (19.7 ppm, 19.9 ppm, 25.7 ppm, 25.9 ppm), while showed $\mathrm{C}=\mathrm{O}$ signals at $182.3 \mathrm{ppm}$. The structure of $\mathbf{2 t}$ was further verified by mass spectroscopy that showed a molecular ion peak $[\mathrm{M}+\mathrm{H}]^{+}$at ESI-HRMS 437.1864 $(55.4 \%)$ in accordance with the molecular formula $\mathrm{C}_{26} \mathrm{H}_{28} \mathrm{O}_{6}$.

\section{Antibacterial activity}

In this study, antibacterial activity was determined from the MIC with different strains in vitro, including multidrug-resistant clinical isolates. Norfloxacin was used as a positive control for bacteria. As shown in Table I, compounds $\mathbf{4 a}-\mathbf{4 t}$ did not exhibit antibacterial activity against Gram-negative strains at a dose of $24-164 \mu \mathrm{M}$ in vitro, but some compounds displayed potent antibacterial activity against Gram-positive strains. Nine compounds gave MIC values of 4.4-19 $\mu \mathrm{M}$. Compounds $\mathbf{4 c}, \mathbf{4 g}, \mathbf{4 i}$, $\mathbf{4 j}, \mathbf{4 k}, \mathbf{4}, \mathbf{4 n}, \mathbf{4 q}$ and $\mathbf{4 t}$ were highly active against $S$. aureus (S. aureus RN4220, S. aureus KCTC 503, and $S$. aureus KCTC 209) and Streptococcus mutans KCTC (S. mutans KCTC 3065 and S. mutans KCTC 3289) strains, with MIC values of 4.4-19 $\mu \mathrm{M}$, but were less active than 
standard drug norfloxacin. The synthesized derivatives showed significant antibacterial effects against $S$. aureus, with 2,4- $\mathrm{Cl}_{2}$ substituted compound $\mathbf{4 k}$ giving a MIC value of $4.4 \mu \mathrm{M}$, which was similarly active to standard drug norfloxacin.

By analyzing the activities of synthesized compounds 4a-4t, the following structure activity relationships were observed. Eight electron-donor compounds including $o-\mathrm{CH}_{3}, m-\mathrm{CH}_{3}, p-\mathrm{CH}_{3}, o-\mathrm{OCH}_{3}, m-\mathrm{OCH}_{3}, p-\mathrm{OCH}_{3}$, $p-\mathrm{N}\left(\mathrm{CH}_{3}\right)_{2}$, and $3-\mathrm{OCH}_{3}-4-\mathrm{OH}$ on the substituent of phenyl ring, were designed and synthesized. Pharmacological test results showed that their activities were lower than those of halogen-substituted derivatives of phenyl ring, with activities in the order $3-\mathrm{OCH}_{3}-4-\mathrm{OH}>m-\mathrm{OCH}_{3}>$ $m-\mathrm{CH}_{3}>\mathrm{H}>o-\mathrm{CH}_{3}, p-\mathrm{CH}_{3}>o-\mathrm{OCH}_{3}, p-\mathrm{OCH}_{3}, p-\mathrm{N}\left(\mathrm{CH}_{3}\right)_{2}$. For different position methyl and methoxy groups on the substituent of phenyl ring influenced the antibacterial effects with activities in the order $m-\mathrm{CH}_{3}>o-\mathrm{CH}_{3}, p-\mathrm{CH}_{3}$, and $m-\mathrm{OCH}_{3}>o-\mathrm{OCH}_{3}, p-\mathrm{OCH}_{3}$. Furthermore, the position of electron-withdrawing $(\mathrm{F}, \mathrm{Cl}$, and $\mathrm{Br})$ groups on the $\mathrm{B}$ ring (of phenyl ring) significantly influenced the antibacterial activity, with activities in the order $m-\mathrm{F}>p-\mathrm{F}>o-\mathrm{F}$ for fluoro-substituted compounds of phenyl ring, and $p-\mathrm{Br}>$ $m-\mathrm{Br}>o-\mathrm{Br}$ for bromo-substituted compounds of phenyl ring. In comparison, the chloro-substituted derivatives of phenyl ring showed activities in the order 2,4-dichloro $>$ $p-\mathrm{Cl}>m-\mathrm{Cl}>o-\mathrm{Cl}$. Therefore, compounds $\mathbf{4} \mathbf{c}, \mathbf{4 g}$, and $\mathbf{4 j}$ bearing $m-\mathrm{F}, p-\mathrm{Cl}$, and $p$ - $\mathrm{Br}$ substituents of phenyl ring, respectively, showed better activities, while those bearing $o-\mathrm{F}, o-\mathrm{Cl}$, and $o-\mathrm{Br}$ substituents of phenyl ring $(4 \mathbf{b}, 4 \mathbf{e}$, and $\mathbf{4 f}$, respectively) were inactive for all microorganisms, even at doses of 24-151 $\mu \mathrm{M}$. Compound $4 \mathbf{k}(\mathrm{MIC}=4.4$ $\mu \mathrm{M})$ was 30 -fold more potent than apigenin $(\mathrm{MIC}=118.5$ $\mu \mathrm{M})$. It has been proposed that the prenyl moiety on A ring

TABLE I - Inhibitory activity of compounds $\mathbf{4 a - 4 t ~ e x p r e s s e d ~ a s ~ M I C ~}(\mu \mathrm{M})$

\begin{tabular}{|c|c|c|c|c|c|c|c|}
\hline \multirow{2}{*}{ Compounds } & \multicolumn{3}{|c|}{ S. aureus } & \multicolumn{2}{|c|}{ S. mutans } & \multicolumn{2}{|c|}{ E. coli } \\
\hline & 4220 & 503 & 209 & 3065 & 3289 & 1924 & 1356 \\
\hline apigenin & 118.5 & 118.5 & 118.5 & 118.5 & 118.5 & $>237$ & $>237$ \\
\hline $4 a$ & 82 & 82 & 82 & 164 & 164 & $>164$ & $>164$ \\
\hline $4 b$ & $>24$ & $>24$ & $>24$ & $>24$ & $>24$ & $>24$ & $>24$ \\
\hline $4 c$ & 9.8 & 9.8 & 9.8 & 9.8 & 9.8 & $>24$ & $>24$ \\
\hline 4d & 24 & 24 & 24 & 24 & 24 & $>24$ & $>24$ \\
\hline $4 e$ & $>151$ & $>151$ & $>151$ & $>151$ & $>151$ & $>151$ & $>151$ \\
\hline $4 f$ & 151 & 151 & 151 & 151 & 151 & $>151$ & $>151$ \\
\hline $4 g$ & 9.4 & 9.4 & 9.4 & 9.4 & 9.4 & $>151$ & $>151$ \\
\hline $4 h$ & $>136.8$ & $>136.8$ & $>136.8$ & $>136.8$ & $>136.8$ & $>136.8$ & $>136.8$ \\
\hline $4 i$ & 17 & 17 & 17 & 17 & 17 & $>136.8$ & $>136.8$ \\
\hline $4 j$ & 8.5 & 8.5 & 8.5 & 8.5 & 8.5 & $>136.8$ & $>136.8$ \\
\hline $4 k$ & 4.4 & 4.4 & 4.4 & 4.4 & 4.4 & $>139.7$ & $>139.7$ \\
\hline 41 & 9.2 & 9.2 & 9.2 & 18.4 & 18.4 & $>147$ & $>147$ \\
\hline $4 m$ & 158 & 158 & 158 & 158 & 158 & $>158$ & $>158$ \\
\hline $4 n$ & 19.8 & 19.8 & 19.8 & 19.8 & 19.8 & $>158$ & $>158$ \\
\hline 40 & 158 & 158 & 158 & 158 & 158 & $>158$ & $>158$ \\
\hline $4 p$ & $>152$ & $>152$ & $>152$ & $>152$ & $>152$ & $>152$ & $>>152$ \\
\hline $4 q$ & 19 & 19 & 19 & 19 & 19 & $>152$ & $>>152$ \\
\hline $4 r$ & $>152$ & $>152$ & $>152$ & $>152$ & $>152$ & $>152$ & $>>152$ \\
\hline $4 s$ & $>147.8$ & $>147.8$ & $>147.8$ & $>147.8$ & $>147.8$ & $>147.8$ & $>147.8$ \\
\hline $4 t$ & 9 & 9 & 9 & 9 & 9 & $>146.8$ & $>146.8$ \\
\hline Norfloxacin & 6 & 6 & 12 & 3 & 3 & 50 & 50 \\
\hline
\end{tabular}

S. aureus RN4220, Staphylococcus aureus RN4220; S. aureus 503, Staphylococcus aureus 503; S. aureus 209, Staphylococcus aureus 209; S. mutans 3065, Streptococus mutans 3065; S. mutans 3289, Streptococus mutans 3289; E. coli 1924, Escherichia coli CCARM 1924; E. coli 1356, Escherichia coli CCARM 1356. 
makes compounds more lipophilic, which leads to a higher affinity with cell membranes, with prenylation shown to afford flavonoids with enhanced antibacterial activities (Ei-Bassuony, Abouzid, 2010; Yu et al., 2015).

\section{Activity against clinical isolates of multidrug- resistant Gram-positive bacteria}

The most active compounds, $\mathbf{4 c}, \mathbf{4 g}, \mathbf{4 i}, \mathbf{4 j}, \mathbf{4 k}, \mathbf{4 l}$, $\mathbf{4 n}, \mathbf{4 q}$ and $\mathbf{4 t}$, were also evaluated for antibacterial effects against clinical isolates of multidrug-resistant Grampositive bacteria (Table II). These derivatives were found to be highly active against these clinical isolates, giving MIC values of 4.0-20 $\mu \mathrm{M}$. Compound $4 \mathbf{k}$ was more potent than norfloxacin against most microorganisms tested, giving an MIC value of $4.0 \mu \mathrm{M}$. This suggested that the introduction of two halogen atoms of phenyl ring and a prenyl moiety on A ring into the hybrid compound played an important role in improving the antibacterial properties (Chen et al., 2014; Marín, Máñez, 2013). Therefore, compound $4 \mathbf{k}$ should be used as the lead compound for further design and investigations.

TABLE II - MIC values (in $\mu \mathrm{M}$ ) against clinical isolates of multidrug-resistant Gram-positive bacterial strains

\begin{tabular}{lccccc}
\hline \multirow{2}{*}{ Compounds } & \multicolumn{2}{c}{ MRSA } & & \multicolumn{2}{c}{ QRSA } \\
\cline { 2 - 3 } \cline { 5 - 6 } $\mathbf{4 c}$ & $\mathbf{3 1 6 7}$ & $\mathbf{3 5 0 6}$ & & $\mathbf{3 5 0 5}$ & $\mathbf{3 5 1 9}$ \\
$\mathbf{4 g}$ & 10 & 10 & & 10 & 10 \\
$\mathbf{4 i}$ & 9.0 & 9.0 & & 9.0 & 9.0 \\
$\mathbf{4 j}$ & 17 & 17 & & 17 & 17 \\
$\mathbf{4 k}$ & 8.5 & 8.5 & & 8.5 & 8.5 \\
$\mathbf{4 l}$ & 4.0 & 4.0 & & 4.0 & 4.0 \\
$\mathbf{4 n}$ & 9.2 & 9.2 & & 9.2 & 9.2 \\
$\mathbf{4 q}$ & 20 & 20 & & 20 & 20 \\
$\mathbf{4 t}$ & 19 & 19 & & 19 & 19 \\
Norfloxacin & 9.1 & 9.1 & & 9.1 & 9.1 \\
\hline
\end{tabular}

MRSA 3167, methicillin-resistant $S$. aureus CCARM 3167; MRSA 3506, methicillinresistant, $S$. aureus CCARM 3506; QRSA 3505, quinolone-resistant $S$. aureus CCARM 3505; QRSA 3519, quinolone-resistant $S$. aureus CCARM 3519

\section{CONCLUSION}

We synthesized a series of novel 5,7-diisoprenyloxyflavone derivatives and evaluated their antibacterial effects against Gram-positive and Gram-negative bacteria.
Compounds 4c, 4g, 4i, 4j, 4k, 4l, 4n, 4q and 4t were highly active against $S$. aureus and $S$. mutans KCTC, giving MIC values of 4.0-20 $\mu \mathrm{M}$, and also showed high activities against clinical isolates of multidrug-resistant Gram-positive bacteria, with MIC values of 4.0-20 $\mu \mathrm{M}$. In particular, compound $\mathbf{4} \mathbf{k}$ was more potent than norfloxacin against most microorganisms tested, giving a better MIC value of $4.0 \mu \mathrm{M}$. This indicated that hybrid compounds possessing flavone and prenyl moieties might possess improved antibacterial properties. These results indicate that the further design and development of such compounds will be of interest in future research.

\section{ACKNOWLEDGEMENTS}

This work was supported by the Science and Technology Program Project of Zhoushan City of China (No. 2016C41005) and the National Natural Science Foundation of China (No. 81560149) and 13th Five-Year Key Projects of Jilin Provincial Education Department of China (No. JJKH2016261H). We also thank Simon Partridge, $\mathrm{PhD}$, from Liwen Bianji, Edanz Editing China (www.liwenbianji.cn/ac), for editing the English text of a draft of this manuscript.

\section{REFERENCES}

Ahmed D, Khan MM, Saeed R. Comparative analysis of phenolics, flavonoids, and antioxidant and antibacterial potential of methanolic, hexanic and aqueous extracts from adiantum caudatum leaves. Antioxidants (Basel). 2015;4(2):394-409.

Banerjee K, Banerjee S, Das S, Mandal M. Probing the potential of apigenin liposomes in enhancing bacterial membrane perturbation and integrity loss. J Colloid Interf Sci. 2015;453:4859.

Chen ZH, Zheng CJ, Sun LP, Piao HR. Synthesis of new chalcone derivatives containing a rhodanine-3-acetic acid moiety with potential anti-bacterial activity. Eur J Med Chem. 2010;45(12):5739-5743.

Chen X, Mukwaya E, Wong MS, Zhang Y. A systematic review on biological activities of prenylated flavonoids. Pharm Biol. 2014;52(5):655-660 .

Choi AY, Choi JH, Lee JY, Yoon KS, Choe W, Ha J, Yeo EJ, Kang I. Apigenin protects HT22 murine hippocampal neuronal cells against endoplasmic reticulum stress-induced apoptosis. Neurochem Int. 2010;57(2):143-152. 
Coelho AL, Vasconcellos MLAA, Simas ABC, Rabi JA, Costa PRR. A convenient synthesis of $( \pm)$-4-Prenylpterocarpin. Synthesis. 1992;10:914-916.

Ei-Bassuony A, Abouzid S. A new prenylated flavanoid with antibacterial activity from propolis collected in Egypt. Nat Prod Commun. 2010;5(1):43-45.

Eumkeb G, Chukrathok S. Synergistic activity and mechanism of action of ceftazidime and apigenin combination against ceftazidime-resistant Enterobacter cloacae. Phytomedicine. 2013;20(3-4):262-269.

Fan X, Reichling J, Wink M. Antibacterial activity of the recombinant antimicrobial peptide Ib-AMP4 from Impatiens balsamina and its synergy with other antimicrobial agents against drug resistant bacteria. Pharmazie. 2013;68(7):628-630.

Friedman M. Overview of antibacterial, antitoxin, antiviral, and antifungal activities of tea flavonoids and teas. Mol Nutr Food Res. 2007;51(1):116-134.

Guan LP, Zhao DH, Chang Y, Sun Y, Ding XL, Jiang JF. Design, synthesis and antidepressant activity evaluation 2'-hydroxy4',6'-diisoprenyloxychalcone derivatives. Med Chem Res. 2013;22(11):5218-5226.

Harbme JB, Williams CA. Advances in flavonoid research since 1992. Phytochem. 2000;55(6):481-504.

Hosseinkhani F, Jabalameli F, Banar M, Abdellahi N, Taherikalani M, Leeuwen WB, Emaneini M. Monoterpene isolated from the essential oil of trachyspermum ammi is cytotoxic to multidrug-resistant pseudomonas aeruginosa and staphylococcus aureus strains. Rev Soc Bras Med Trop. 2016;49(2):172-176.

Karakaya S, Sedef NEL. Quercetin, luteolin, apigenin and kaempferol contents of some foods. Food Chem. 1999;66(3):289-292.

Keshari AK, Kumar G, Kushwaha PS, Bhardwaj M, Kumar P, Rawat A, Kumar D, Prakash A, Ghosh B, Saha S. Isolated flavonoids from Ficus racemosa stem bark possess antidiabetic, hypolipidemic and protective effects in albino Wistar rats. J Ethnopharmacol. 2016;181:252-262.

Kim S, Sohn DW, Kim YC, Kim SA, Lee SK, Kim HS. Fine tuning of a reported synthetic route for biologically active flavonoid, baicalein. Arch Pharm Res. 2007;30(1):18-21.
Kozyra M, Biernasiuk A, Malm A, Chowaniec M. Chemical compositions and antibacterial activity of extracts obtained from the inflorescences of Cirsium canum (L.) all. Nat Prod Res. 2015;29(21):2059-2063.

Kulbacka J, Pucek A, Kotulska M, Dubińska-magiera M, Rossowska J, Rols MP, Wilk KA. Electroporation and lipid nanoparticles with cyanine IR-780 and flavonoids as efficient vectors to enhanced drug delivery in colon cancer. Bioelectrochem. 2016;110:19-31.

Liu R, Zhang H, Yuan M, Zhou J, Tu Q, Liu JJ, Wang J. Synthesis and biological evaluation of apigenin derivatives as antibacterial and antiproliferative agents. Molecules. 2013;18(9):11496-11511.

Marín M, Máñez S. Recent trends in the pharmacological activity of isoprenyl phenolics. Curr Med Chem. 2013;20(2):272-279.

Rao GV, Swamy BN, Chandregowda V, Reddy GC. Synthesis of (+/-) Abyssinone I and related compounds:Their anti-oxidant and cytotoxic activities. Eur J Med Chem. 2009;44(5):22392245.

Ruivo J, Francisco C, Oliveira R, Figueiras A. The main potentialities of resveratrol for drug delivery systems. Braz $\mathrm{J}$ Pharm Sci. 2015;51(3):499-513.

Sharma PK, Chandak N, Kumar P, Sharma C, Aneja KR. Synthesis and biological evaluation of some 4-functionalizedpyrazoles as antimicrobial agents. Eur J Med Chem. 2011;46(4):1425-1432.

Song MX, Zheng CJ, Deng XQ, Wang Q, Hou SP, Liu TT, Xing XL, Piao HR. Synthesis and bioactivity evaluation of rhodanine derivatives as potential anti-bacterial agents. Eur J Med Chem. 2012;54:403-412.

Turktekin M, Konac E, Onen HI, Alp E, Yilmaz A, Menevse S. Evaluation of the effects of the flavonoid apigenin on apoptotic pathway gene expression on the colon cancer cell line (HT29). J Med Food. 2011;14(10):1107-1117.

Verlinden BK, Louw A, Birkholtz LM. Resisting resistance: is there a solution for malaria? Expert Opin Drug Discov. 2016;11(4):395-406.

Vogel S, Ohmayer S, Brunner G, Heilmann J. Natural and nonnatural prenylate chalcones: synthesis, cytotoxicity and antioxidative activity. Bioorg Med Chem. 2008;16(8):4286-4293. 
Vogel S, Barbic M, Jürgenliemk G, Heilmann J. Synthesis, cytotoxicity, anti- oxidative and anti-inflammatory activity of chalcones and influence of A-ring modifications on the pharmacological effect. Eur J Med Chem. 2010;45(6):22062213.

Winans KA, King DA, Rao VR, Bertozzi CR. A Chemically synthesized version of the insect antibacterial glycopeptide, diptericin, disrupts bacterial membrane integrity. Biochem. 1999;38(36):11700-11710.

Wang HM, Zhang L, Liu J, Yang ZL, Zhao HY, Yang Y, Shen D, Lu K, Fan ZC, Yao QW, Zhang YM, Teng YO, Peng Y. Synthesis and anticancer activity evaluation of novel prenylated and geranylated chalcone natural products and their analogs. Eur J Med Chem. 2015;92:439-448.

Xie C, Peng Z, Zhao SL, Pan CY, Guan LP, Sun XY. Synthesis of 2 '-hydroxy-4'- isoprenyloxychalcone derivatives with potential antidepressant-like activity. Med Chem. 2014;10(8):789-799.
Yao Y, Lin G, Xie Y, Ma P, Li G, Meng Q, Wu T. Preformulation studies of myricetin: a natural antioxidant flavonoid. Pharmazie. 2014;69(1):19-26.

Yu Q, Ravu RR, Xu QM, Ganji S, Jacob MR, Khan SI, Yu BY, Li XC. Antibacterial prenylated acylphloroglucinols from psorothamnus fremontii. J Nat Prod. 2015;78(11):2748-2753 .

Zhao LM, Jin HS, Wan LJ, Zhang LM. General and highly a-regioselective zinc-mediated prenylation of aldehydes and ketones. J Org Chem. 2011;76(6):1831- 1837.

Zhen XH, Quan YC, Peng Z, Han Y, Zheng ZJ, Guan LP. Design, synthesis, and potential antidepressant-like activity of 7-prenyloxy-2,3-dihydroflavanone derivatives. Chem Biol Drug Des. 2016;87(6):858-866.

Received for publication on $07^{\text {th }}$ November 2017 Accepted for publication on $23^{\text {rd }}$ October 2018 